\title{
Putative mitochondrial $\alpha$-ketoglutarate-dependent dioxygenase Fmp12 controls utilization of proline as an energy source in Saccharomyces cerevisiae
}

\author{
Ikuhisa Nishida, Daisuke Watanabe, and Hiroshi Takagi* \\ Graduate School of Biological Sciences, Nara Institute of Science and Technology, 8916-5 Takayama, Ikoma, Nara 630-0192, Japan. \\ * Corresponding Author: \\ Hiroshi Takagi, Graduate School of Biological Sciences, Nara Institute of Science and Technology; 8916-5 Takayama, Ikoma, Nara 630- \\ 0192; Japan; Tel: +81 74372 5420; Fax: +81 74372 5429; E-mail: hiro@bs.naist.jp
}

\begin{abstract}
The amino acid proline functions as a nitrogen source and as a stress protectant in the yeast Saccharomyces cerevisiae. However, utilization of proline as a carbon source in S. cerevisiae cells has not been studied yet. In the process of study on the physiological roles of the found-in-mitochondrialproteome (FMP) genes in proline metabolism, we found that $\Delta$ fmp12 cells could grow better than wild-type cells on agar plate medium containing proline as the sole nitrogen and carbon sources. In contrast, overexpression of FMP12 negatively affected cell growth under the same condition. The Fmp12 protein was localized in the mitochondria and was constitutively expressed. Deletion of the genes that encode mitochondrial enzymes, such as proline dehydrogenase (PUT1), $\Delta^{1}$-pyrroline-5-carboxylate dehydrogenase (PUT2), alanine transaminase (ALT1), and $\alpha$-ketoglutarate dehydrogenase subunit (KGD1), abolished the enhanced cell growth in $\Delta$ fmp12. These results provided the first evidence that proline can be utilized as a carbon source via the mitochondrial proline metabolic pathway and the subsequent tricarboxylic acid (TCA) cycle in S. cerevisiae. The function of Fmp12, which has a similarity with $\alpha$-ketoglutarate-dependent dioxygenases of the yeast Candida species and human, might inhibit cell growth by skipping the ATP production step of the TCA cycle.
\end{abstract}

doi: 10.15698/mic2016.10.535

Received originally: 12.07.2016;

in revised form: 15.08.2016,

Accepted 19.08.2016,

Published 19.09.2016.

Keywords: yeast Saccharomyces cerevisiae, proline, carbon source, Fmp12, mitochondria, $\alpha$ ketoglutarate-dependent dioxygenase.

\section{Abbreviations:}

$A A T$ - amino acid transaminase, FMP - found-in-mitochondrialproteome, TCA - tricarboxylic acid.

\section{INTRODUCTION}

Proline is an important amino acid that is used not only for a nitrogen source but also a stress protectant in the budding yeast Saccharomyces cerevisiae [1]. Therefore, the metabolic regulation of proline, including biosynthesis, degradation, and cellular localization, has been of great interest. S. cerevisiae cells synthesize proline from glutamate by three cytoplasmic enzymes, the $\gamma$-glutamyl kinase Pro1, the $\gamma$-glutamyl phosphate reductase Pro2, and the $\Delta^{1}$ pyrroline-5-carboxylate (P5C) reductase Pro3 [2]. In most of eukaryote cells, including $S$. cerevisiae, proline is degraded predominantly in mitochondria, although the responsible import mechanism into mitochondria has not been clarified yet. The mitochondrial proline dehydrogenase Put1 converts proline into $\mathrm{P} 5 \mathrm{C}$, which is then processed into glutamate by the P5C dehydrogenase Put2 [3]. As glutamate can be converted into $\alpha$-ketoglutarate, a tricarboxylic acid (TCA)-cycle intermediate, by deamination or transamination $[4,5]$, it is presumed that proline can be used as a carbon source for ATP production. Nevertheless, there have been few reports on the growth of S. cerevisiae cells in the presence of proline or the other amino acids as the sole carbon source. In contrast, several kinds of bacteria and xylose-fermenting yeast Scheffersomyces stipitis have been reported to grow by utilization of amino acids as the sole carbon source via glutamate dehydrogenase or amino acid transaminase (AAT) [6-9]. Based on these facts, $S$. cerevisiae cells might possess an unknown metabolic regulatory mechanism that restricts the consumption of amino acids as carbon sources.

To find novel regulators of intracellular proline, we previously performed a transcriptomic analysis of the S. cerevisiae put1 mutant in response to exogenous proline [10]. As a result, the accumulation of intracellular proline led to downregulation of the proline-synthetic genes and upregulation of the genes that encode the proline-degradative enzymes, the known proline permeases on the plasma membrane, and the Avt proteins associated with bidirec- 


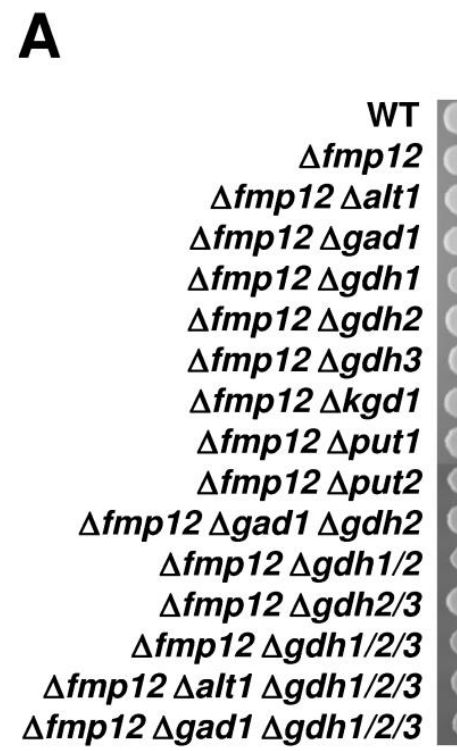

B

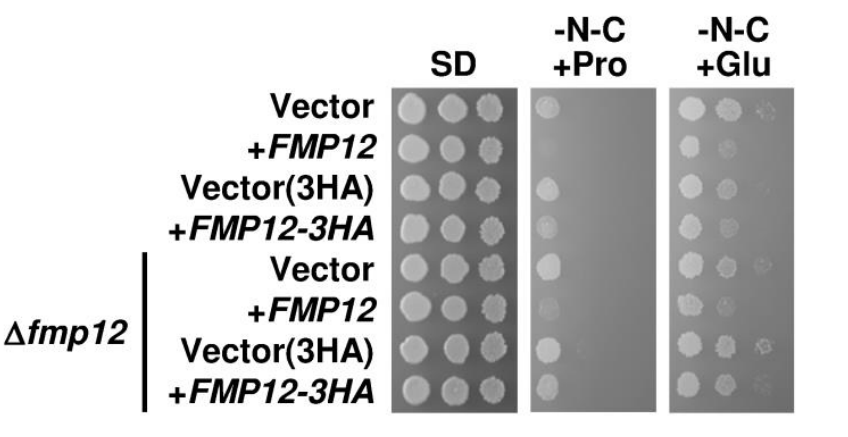

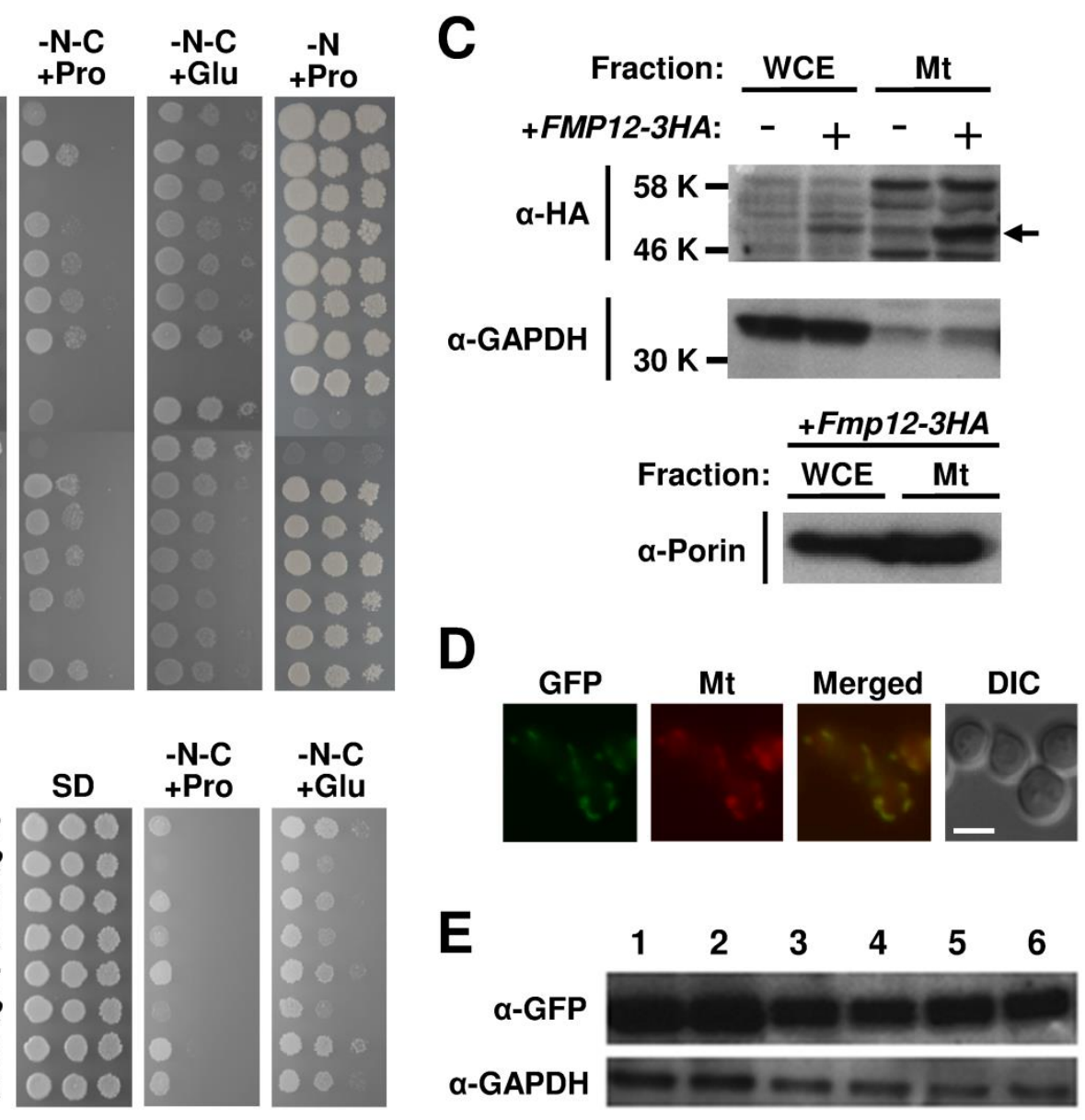

F

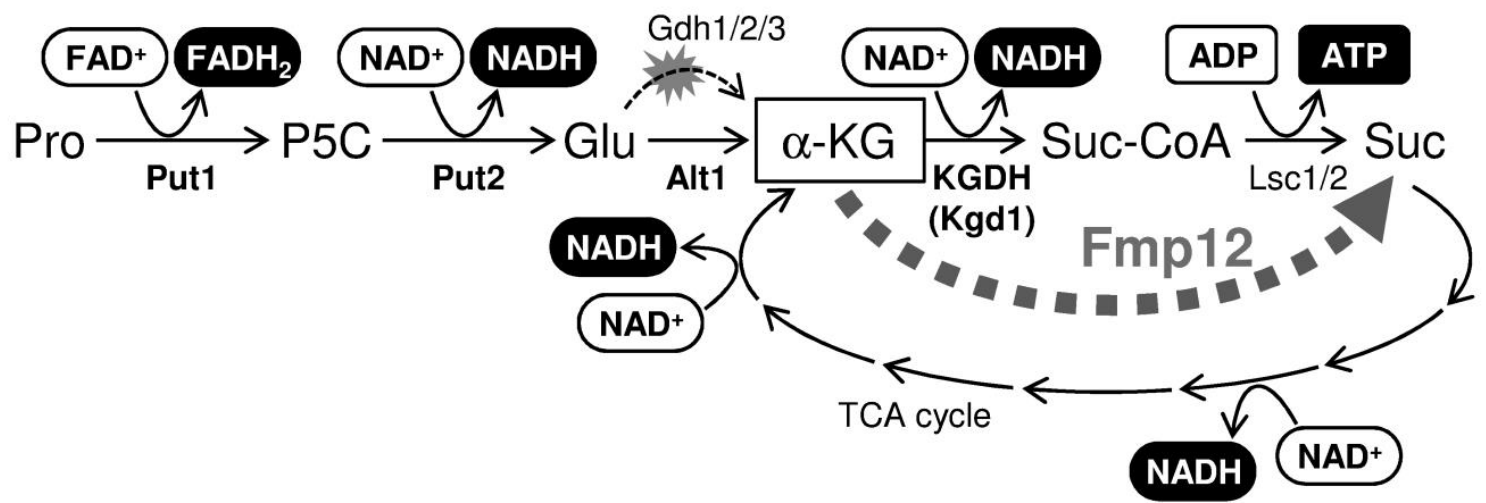

FIGURE 1: Proline/glutamate metabolism as the sole carbon source and its control mediated by Fmp12. (A) Growth phenotypes of S. cerevisiae wild-type strain (BY4741) and its deletion mutants on SD, SD-N-C+Pro, SD-N-C+Glu, and SD-N+Pro media. (B) Growth phenotypes of S. cerevisiae BY4741u or BY4741u $\triangle f m p 12$ cells harboring the empty vector (pVV208, pVV209) or overexpressing FMP12 (pVV208-FMP12, pVV209-FMP12) on $\mathrm{SD}, \mathrm{SD}-\mathrm{N}-\mathrm{C}+$ Pro, and SD-N-C+Glu media. (C) Detection of Fmp12-3HA in the whole cell extract (WCE) and in the mitochondria fraction (Mt). The symbol -/+ indicates the sample from the cells without or with expression of Fmp12-3HA from pVV209-FMP12. The arrow indicates the predicted size of Fmp12-3HA truncated at its amino-terminal MTS. GAPDH and porin were analyzed as controls of cytoplasmic and mitochondrial proteins, respectively. (D) Subcellular localization of Fmp12-yeGFP by fluorescent microscopy. GFP signals (GFP), MitoTracker signals (Mt), merged signals (Merged), and differential interference contrast images (DIC) are shown. The scale bar represents $5 \mu \mathrm{m}$. (E) Western blot analysis of Fmp12-yeGFP under different nutritional conditions. S. cerevisiae FMP12-yeGFP cells were cultivated on solid media at $30^{\circ} \mathrm{C}$ for 5 days. The numbers indicate the media as follows: 1: SD, 2: SD+Pro, 3: SD-N-C+Pro, 4: SD-C+Pro, 5: SD-N-C+Glu, 6: SD-C+Glu. GAPDH was used as a proteinloading control. (F) Energy production by proline metabolism as the sole carbon source in S. cerevisiae. Pro: proline, P5C: $\Delta^{1}$-pyrroline-5carboxylate, Glu: glutamate, $\alpha$-KG: $\alpha$-ketoglutarate, Suc-CoA: succinyl-CoA, Suc: succinate. 
tional transport of proline across the vacuolar membrane. In this study, we analyzed another class of the upregulated genes that encode poorly-understood proteins originally found in the mitochondrial proteome analyses [11, 12], because these genes (the found-in-mitochondrialproteome (FMP) genes) and their products (the Fmp proteins) might be involved in novel regulatory mechanisms of proline localization or metabolism in the mitochondria.

\section{RESULTS}

To examine cell growth in the presence of proline as the sole carbon and nitrogen sources, we used minimal synthetic-defined (SD) medium without ammonium sulfate and glucose and with proline (SD-N-C+Pro). Under such a severe nutrient condition, BY4741 wild-type cells in which the auxotrophic mutations were complemented could only slightly grow during 7-day incubation (Figure 1A). Interestingly, among the FMP genes (FMP12/16/21/27/33/41/45/ $46 / 48 / 52$ ) that were upregulated by exogenous proline in our previous study [10], deletion of the FMP12/AIM17 gene was found to markedly enhance the growth on SD-NC+Pro agar plates (Figure 1A), as well as on SD-C+Pro agar plates, in which proline and ammonium sulfate was added as the sole carbon and nitrogen source, respectively (data not shown). In contrast, overexpression of the FMP12 gene inhibited the growth on SD-N-C+Pro agar plates (Figure 1B) Similar results were obtained from monosodium glutamate as the sole carbon and nitrogen sources (SD-N-C+Glu) instead of proline, although yeast cells grew better in SD-N$\mathrm{C}+\mathrm{Glu}$ than in SD-N-C+Pro. These data suggest the presence of the proline/glutamate metabolic pathway as the sole carbon source in $S$. cerevisiae, although this pathway might be strictly restricted by an unknown mechanism involving Fmp12.

Fmp12 was originally identified in high-throughput mitochondrial proteome analyses $[11,12]$. To determine the subcellular localization of Fmp12, we used HA- or yeGFPtagged Fmp12 at the carboxyl terminus. Overexpression of Fmp12-3HA was shown to inhibit cell growth on SD-N$\mathrm{C}+$ Pro or SD-N-C+Glu medium (Figure $1 \mathrm{~B}$ ), suggesting that carboxyl-terminus tagged Fmp12 proteins partly functions. It is also noted that the growth of Fmp12-yeGFP-expressing cells on SD-N-C+Pro or SD-N-C+Glu medium was comparable to that of wild-type cells (data not shown). In a western blot analysis (Figure 1C), the HA signals dependent on overexpression of Fmp12-3HA were detected at the lower molecular weight than we expected (approximately 58 $\mathrm{kDa}$ ) both in the whole cell extract and in the mitochondrial fraction. This is probably because amino-terminal 46 amino acid residues, which correspond to a mitochondrialtargeting signal (MTS) predicted by MitoProt II analysis (https://ihg.gsf.de/ihg/mitoprot.html), were truncated [13] As shown in Figure 1C, a stronger signal of Fmp12-3HA was observed in the mitochondria fraction, which was indicated by a lower level of the cytoplasmic glyceraldehyde-3phosphate dehydrogenase (GAPDH) and a higher level of the mitochondrial porin protein, than in the whole cell extract. Additionally, the fluorescence microscope observa- tion revealed that Fmp12-yeGFP-derived fluorescent signals colocalized with the MitoTracker signals (Figure 1D). Based on these results, it was concluded that Fmp12 specifically localizes at the mitochondria. The expression level of Fmp12-yeGFP was not significantly affected by exogenous carbon or nitrogen sources (Figure $1 \mathrm{E}$ ), although the FMP12 mRNA was previously found to be upregulated by the addition of proline to the medium [10].

To identify the metabolic pathway of proline as a carbon source in $\Delta f m p 12$ cells, we examined the effects of gene deletions on the growth on SD-N-C+Pro medium (Figure $1 \mathrm{~A}$ ). Enhanced growth of $\Delta f m p 12$ cells was almost fully suppressed by a single disruption of the PUT1 (encoding proline dehydrogenase), PUT2 (encoding P5C dehydrogenase), $A L T 1$ (encoding mitochondrial alanine transaminase) [14], or KGD1 (encoding E1 component of the mitochondrial $\alpha$-ketoglutarate dehydrogenase (KGDH) complex) [15] gene, but not affected by single or combined deletion of the $G D H 2$ (encoding NAD+-dependent glutamate dehydrogenase) and GDH1/3 (encoding $\mathrm{NADP}^{+}$-dependent glutamate dehydrogenases) genes involved in deamination of glutamate and its reverse reaction, respectively [5, 16, 17], or by the GAD1 (encoding glutamate decarboxylase) gene, whose product catalyzes the first step of the $\gamma$ aminobutyrate (GABA) shunt [18] that bypasses the TCA cycle. Similar results were obtained on SD-N-C+Glu medium, although disruption of the PUT1 or PUT2 gene did not abolish the growth of $\Delta f m p 12$ cells. Thus, it was demonstrated that utilization of proline as a carbon source is specifically carried out by mitochondrial enzymes Put1 and Put2 for conversion into glutamate, by mitochondrial AATs, such as the alanine transaminase Alt1, for conversion of glutamate into $\alpha$-ketoglutarate, and subsequently by the TCA cycle (Figure 1F). Since Put1 and Put2, but not Alt1 and Kgd1, are necessary for utilization of proline as a nitrogen source judging from the growth on SD-N+Pro medium (Figure $1 \mathrm{~A}), \mathrm{AATs}$ and $\mathrm{KGDH}$ were identified as specific factors required for utilization of proline as the sole carbon source.

\section{DISCUSSION}

Compartmentalization of metabolic enzymes might contribute to optimization of the corresponding metabolic efficiency. If the enzymes with different subcellular localizations are engaged in a metabolic pathway, intermediate compounds must be properly translocated to the sites or the organelles in advance to individual reactions. Although cells might adopt such spatial regulations to constitute highly elaborate metabolic networks, enforcement of a single metabolic pathway is likely achieved by collecting all related enzymes together in a single intracellular area. For instance, it was recently reported that artificial overexpression of the Ehrlich pathway enzymes in mitochondria led to a striking increase in the fusel alcohol production in $S$. cerevisiae [19].

In this study, we discovered a novel proline metabolic pathway essentially mediated by the mitochondrial enzymes Put1, Put2, Alt1, KGDH, and TCA-cycle enzymes (Figure $1 \mathrm{~F})$. This compartmentalization might maximize cellular 
energy production from proline under nutrient-poor environments. The major problem to be solved is how proline is imported into mitochondria. Effects of the mitochondrial transporters on subcellular distribution of proline should be biochemically analyzed in future. It is also noteworthy that the mitochondrial alanine transaminase Alt1 is used for the conversion of glutamate into $\alpha$-ketoglutarate in $S$. cerevisiae, while glutamate dehydrogenases are responsible in known proline utilization pathways of other yeast species [8]. Proline utilization via the alanine transaminase activity has been reported only in parasitic protozoan Trypanosoma brucei [20] and tsetse fly [21], which might exhibit similar physiological and nutritional conditions as $S$. cerevisiae cells when proline is the primary carbon source.

To understand how the deletion of FMP12 enhances cell growth on SD-N-C+Pro medium, we further focused on its molecular function. The Fmp12 protein shows a marked similarity to $\alpha$-ketoglutarate-dependent dioxygenases conserved from bacteria to human [22], such as the pathogenic yeast Candida albicans $\gamma$-butyrobetaine dioxygenase Bbh1 and trimethyllysine dioxygenase Bbh2 mediating the carnitine biosynthesis pathway [23]. They commonly couple decarboxylation of $\alpha$-ketoglutarate into succinate to hydroxylation of numerous kinds of cosubstrates (e.g. Ybutyrobetaine and trimethyllysine). Although the specific substrate for Fmp12 has not been known yet, the Fmp12dependent decarboxylation of $\alpha$-ketoglutarate might enable to bypass the TCA-cycle reactions mediated by KGDH (from $\alpha$-ketoglutarate to succinyl-CoA) and succinyl-CoA ligase (from succinyl-CoA to succinate) (Figure 1F). If $\alpha$ ketoglutarate prefers Fmp12 to $\mathrm{KGDH}$, however, $\mathrm{NADH}$ production via $\mathrm{KGDH}$ and ATP production via succinyl-CoA ligase might be skipped. In particular, when proline is the sole carbon source, the only substrate-level phosphorylation process for ATP production might be impaired by the Fmp12 activity. Therefore, the deletion of FMP12 might enhance energy production and cell growth on SD-N-C+Pro medium. To prove this hypothesis, the affinity of Fmp12 or KGDH for $\alpha$-ketoglutarate should be enzymatically determined. If this is the case, it could be concluded that Fmp12 plays a critical inhibitory role in utilization of amino acids as a carbon source. Nutrient preferences of $S$. cerevisiae cells are strictly determined by several mechanisms including carbon/nitrogen catabolite repression [24, 25]. Analysis of this novel Fmp12 protein will accelerate the understanding of the coordinated mechanism of nutrient sensing and growth control in S. cerevisiae.

\section{MATERIALS AND METHODS \\ Strains}

Yeast strains used in this study were the $S$. cerevisiae strains with a BY4741 (MATa his $3 \Delta 1$ leu2 $\Delta 0$ met15 10 ura3 $\Delta 0$ ) background (provided by Open Biosystems or National BioResource Project (NBRP) of the MEXT, Japan) and BY4741u (MATa ura $3 \triangle 0$ ) background (constructed by $\mathrm{S}$. Morigasaki). The yeast strains used in this study are listed in Table 1. In strain BY4741, the original genes on the chromosomes were disrupted by a standard PCR-based gene disruption or tagging method. Disruption cassettes amplified by PCR with pFA6a-
GFP(S65T)-His3MX6 (Addgene), pFA6a-hphNT1 (Euroscarf), pFA6a-natNT2 (Euroscarf), or an S1-AUR1-S2 fragment derived from pAUR123 (Takara Bio) using gene-specific oligonucleotide primers were integrated into each locus of the target genes. Fmp12 was carboxyl terminally-tagged with yeGFP using pYM25 (Euroscarf). Gene disruptants were selected on YPD medium containing G418 $(200 \mu \mathrm{g} / \mathrm{ml})$, clonNAT (100 $\mu \mathrm{g} / \mathrm{ml})$, hygromycin B $(300 \mu \mathrm{g} / \mathrm{ml})$, or aureobasidin A $(0.25$ $\mu \mathrm{g} / \mathrm{ml}$ ). SC-His medium was used to obtain strains disrupted with CgHIS3 containing disruption cassettes. Correct disruption was confirmed by colony PCR using up and down primers of the target genes. When appropriate, required empty vectors pAD4 (provided by J. Nikawa), pRS416MET15 (constructed by S. Morigasaki), pRS416CgHIS3MET15 (constructed by S. Morigasaki) were introduced to complement the auxotrophy. E. coli strains $\mathrm{DH} 5 \alpha$ (F- $\lambda$ - $\Phi 80$ lacZ $\triangle M 15 \Delta$ (lacZYA argF)U169 deoR recA1 endA1 $h s d R 17\left(r_{k}^{-} m_{k}^{+}\right)$supE44 thi-1 gyrA96) and DB3.1 (F- gyrA462 endA1 $\Delta$ (sr1-recA) $m c r B ~ m r r ~ h s d S 2 O\left(\mathrm{r}_{\mathrm{B}^{-}} \mathrm{m}_{\mathrm{B}}{ }^{-}\right.$) supE44 ara-14 galK2 lacY1 proA2 rpsL2O(SmR) xyl-5 $\lambda$ - leu $m t / 1$ ) were used for construction of the plasmids.

\section{Plasmids}

For overexpression of Fmp12, we constructed plasmids containing a Tet-Off system [26]. BP and LR recombination reaction procedures were performed as recommended by Invitrogen (on the Internet at www.invitrogen.com). Plasmid BG1805-FMP12 (Addgene) containing oligonucleotides of FMP12 without stop codon flanked with attB1 or attB2 sequences was cloned into pDONR221 (Invitrogen) using the BP reaction. The cloned fragment was then inserted into the empty vector pVV208 (CEN URA3 pTetO7) or pVV209 (CEN URA3 pTetO7 3HA) [26] by the LR reaction.

\section{Culture Media}

The media used for growth of $S$. cerevisiae were a nutrient medium YPD ( $1 \%$ yeast extract, $2 \%$ peptone, and $2 \%$ glucose) and a synthetic minimal medium SD $(0.17 \%$ yeast nitrogen base without amino acids and ammonium sulfate (Difco Laboratories), $0.5 \%$ ammonium sulfate, and $2 \%$ glucose). When appropriate, $3 \%$ proline or $3 \%$ monosodium glutamate were added to the media as the sole carbon and nitrogen source. For SD-N+Pro medium, $0.1 \%$ proline was added instead of ammonium. If necessary, $2 \%$ agar was added to solidify the medium. All amino acids used in this study are L-form. E. coli cells were grown in Luria-Bertani (LB) complete medium $0.5 \%$ Bacto yeast extract, $1 \%$ Bacto tryptone (Difco Laboratories), $1 \% \mathrm{NaCl}, \mathrm{pH} 7.0$ ) with $100 \mu \mathrm{g} / \mathrm{ml}$ ampicillin or $50 \mu \mathrm{g} / \mathrm{ml}$ kanamycin.

\section{Growth Test}

Fresh yeast cells were cultured in SD liquid medium for 1 day at $30^{\circ} \mathrm{C}$. After washing the cells with distilled water three times, cells corresponding to an $\mathrm{OD}_{600}$ of 2.0 and serial dilution of $10^{-1}$ to $10^{-3}$ were spotted onto the agar plates and were incubated at $30^{\circ} \mathrm{C}$ for several days.

\section{Fluorescent Microscopy}

Yeast cells expressing Fmp12-yeGFP were grown to reach an $\mathrm{OD}_{600}$ of 1 at $25^{\circ} \mathrm{C}$ in SD liquid medium and subjected to $40 \mathrm{nM}$ MitoTracker Orange CMTMRos (Invitrogen) for $30 \mathrm{~min}$ to visualize the mitochondria. The cells were then harvested and washed with distilled water. Fmp12-yeGFP and MitoTracker 
TABLE 1. Yeast strains in this study.

\begin{tabular}{|c|c|}
\hline Strain & Genotype \\
\hline BY4741 & MATa his $3 \Delta 1$ leu $2 \Delta 0$ met $15 \Delta 0$ ura $3 \Delta 0$ \\
\hline$\Delta f m p 12$ & BY4741 $\Delta$ fmp12::kanMX4 \\
\hline$\Delta f m p 12 \Delta a l t 1$ & BY4741 $\Delta f m p 12:: k a n M X 4 \Delta a l t 1:: h p h N T 1$ \\
\hline$\Delta f m p 12 \Delta g a d 1$ & BY4741 $\Delta$ fmp12::kanMX4 $\Delta$ gad1::hphNT1 \\
\hline$\Delta f m p 12 \Delta g d h 1$ & BY4741 $\Delta f m p 12:: k a n M X 4 \Delta g d h 1:: h p h N T 1$ \\
\hline$\Delta f m p 12 \Delta g d h 2$ & BY4741 $\Delta$ fmp12::kanMX4 $\Delta g d h 2:: h p h N T 1$ \\
\hline$\Delta f m p 12 \Delta g d h 3$ & BY4741 $\Delta f m p 12:: k a n M X 4 \Delta g d h 3:: h p h N T 1$ \\
\hline$\Delta f m p 12 \Delta k g d 1$ & BY4741 $\Delta f m p 12:: k a n M X 4 \Delta k g d 1:: h p h N T 1$ \\
\hline$\Delta f m p 12 \Delta p u t 1$ & BY4741 $\Delta f m p 12:: k a n M X 4 \Delta p u t 1:: h p h N T 1$ \\
\hline$\Delta f m p 12 \Delta p u t 2$ & BY4741 $\Delta f m p 12:: k a n M X 4 \Delta p u t 2:: h p h N T 1$ \\
\hline$\Delta f m p 12 \Delta g a d 1 \Delta g d h 2$ & BY4741 $\Delta$ fmp12::kanMX4 $\Delta$ gad1::hphNT1 $\Delta g d h 2:: n a t N T 2$ \\
\hline$\Delta f m p 12 \Delta g d h 1 / 2$ & BY4741 $\Delta$ fmp12::kanMX4 $\Delta g d h 1:: h p h N T 1 \Delta g d h 2:: n a t N T 2$ \\
\hline$\Delta f m p 12 \Delta g d h 2 / 3$ & BY4741 $\Delta$ fmp12::kanMX4 $\Delta g d h 2:: n a t N T 2 \Delta g d h 3:: h p h N T 1$ \\
\hline$\Delta f m p 12 \Delta g d h 1 / 2 / 3$ & BY4741 $\Delta$ fmp12::kanMX4 $\Delta g d h 1:: A U R 1-C \Delta g d h 2:: n a t N T 2 \Delta g d h 3:: h p h N T 1$ \\
\hline$\Delta f m p 12 \Delta a l t 1 \Delta g d h 1 / 2 / 3$ & BY4741 $\Delta f m p 12:: k a n M X 4$ Aalt1::HISMX6 $\Delta g d h 1:: A U R 1-C \Delta g d h 2:: n a t N T 2 \Delta g d h 3:: h p h N T 1$ \\
\hline$\Delta f m p 12 \Delta g a d 1 \Delta g d h 1 / 2 / 3$ & BY4741 $\Delta$ fmp12::kanMX4 $\Delta g a d 1:: H I S M X 6 \Delta g d h 1:: A U R 1-C \Delta g d h 2:: n a t N T 2 \Delta g d h 3:: h p h N T 1$ \\
\hline Fmp12-yeGFP & BY4741 FMP12::FMP12-yeGFP-hphNT1 \\
\hline BY4741u & MATa ura3 $\triangle 0$ \\
\hline BY4741u $\Delta f m p 12$ & MATa ura3 $\triangle 0 \triangle$ fmp12::kanMX4 \\
\hline
\end{tabular}

signals were observed under a fluorescence microscope Axiovert 200M (Carl Zeiss). Images were captured with a HBO 100 Microscope Illuminating System (Carl Zeiss) digital camera.

\section{Western Blotting Analysis}

Yeast cells grown on agar plates for 5 days at $30^{\circ} \mathrm{C}$ were collected, washed with $10 \%$ trichloroacetic acid (TCA), resuspended by $100 \mu \mathrm{l}$ of $10 \% \mathrm{TCA}$, and disrupted with $0.5-\mathrm{mm}$ glass beads in a MultiBead Shocker (Yasui Kikai) with seven cycles of running at 2,500 rpm for 60 seconds and pausing for 60 seconds. After adding $200 \mu \mathrm{l}$ of $10 \%$ TCA to the tube, the pellets were separated from the glass beads by centrifugation. The obtained pellets were resuspended in $150 \mu \mathrm{L}$ of sample buffer ( $50 \mathrm{mM}$ Tris- $\mathrm{HCl}$ ( $\mathrm{pH} 6.8$ ), $2 \%$ sodium dodecyl sulfate, $4.5 \%$ glycerol, $0.01 \%$ bromophenol blue, $0.7 \mathrm{M}$ 2mercaptoethanol) and $150 \mu \mathrm{l}$ of $1 \mathrm{M}$ Tris- $\mathrm{HCl}(\mathrm{pH} 8.0)$ for neutralization. After boiling for $5 \mathrm{~min}$ at $95^{\circ} \mathrm{C}$, the supernatant was obtained by centrifugation and the protein concentration was measured by using a Bio-Rad protein assay kit (Bio-Rad). The mitochondrial fraction was isolated according to the method described previously [27] with minor modification. In the case of the Fmp12-3HA protein, lysates of yeast cells grown in lactate minimal medium $(0.17 \%$ Bacto yeast nitrogen base without amino acids and ammonium sulphate (Difco Laboratories), $0.5 \%$ ammonium sulphate, $2 \%$ dropout mix, $0.05 \%$ glucose, $0.05 \% \mathrm{CaCl}_{2}, 0.05 \% \mathrm{NaCl}, 0.03 \% \mathrm{MgCl}_{2}, 0.1 \%$ $\mathrm{KH}_{2} \mathrm{PO}_{4}, 2 \%$ lactate $\left.(\mathrm{pH} 5.6)\right)$ or the mitochondrial fractions were directly mixed with sample buffer and boiled. Approximately $5 \mu \mathrm{g}$ of total proteins were loaded onto a 10\% SDSpolyacrylamide gel and electrophoresed with a constant voltage at $80-120 \mathrm{~V}$. Proteins were transferred to a nitrocellulose membrane (Hybond-C; GE Healthcare) in blotting buffer (48 $\mathrm{mM}$ Tris, $385 \mathrm{mM}$ glycine, 0.1\% SDS, 20\% methanol) (115 V, $400 \mathrm{~mA}, 1 \mathrm{~h}$ ). The membrane was soaked in blocking buffer (Tris-buffered saline with 0.3\% Tween-20, 3\% skim milk, 0.03\% sodium azide $(\mathrm{pH} 8.0))$ for more than $1 \mathrm{~h}$. Anti-GFP mouse 
monoclonal (Roche Diagnostics), anti-HA rabbit polyclonal (Santa Cruz Biotechnology), anti-yeast GAPDH rabbit polyclonal (Nordic Immunology) and anti-yeast porin mouse monoclonal (Invitrogen) antibodies were used as primary antibodies, and horseradish peroxidase-fused anti-mouse or anti-rabbit immunoglobulin G (Promega) were used as a secondary antibody. Detection was performed using the Pierce ECL Plus Western Blotting Substrate (Thermo Fisher).

\section{ACKNOWLEDGMENTS}

We thank Dr. Junichi Nikawa, Dr. Susumu Morigasaki, and the National BioResource Project (NBRP), Japan for providing plasmids and yeast strains. This work was supported in part by Grant-in-Aid for JSPS Fellows (11J07980) to I.N. and the Asahi Group Sciences Foundation (Life Science field, 2013) to H.T.

\section{REFERENCES}

1. Takagi H (2008). Proline as a stress protectant in yeast: physiological functions, metabolic regulations, and biotechnological applications. Appl Microbiol Biotech 81(2): 211-223.

2. Tomenchok DM and Brandriss MC (1987). Gene-enzyme relationships in the proline biosynthetic pathway of Saccharomyces cerevisiae. J Bacteriol 169(12): 5364-5372.

3. Brandriss MC and Magasanik B (1979). Genetics and physiology of proline utilization in Saccharomyces cerevisiae: enzyme induction by proline. J Bacteriol 140(2): 498-503.

4. Green DE, Leloir LF, and Nocito V (1945). Transaminases. J Biol Chem 161: 559-582.

5. Miller SM and Magasanik B (1990). Role of NAD-linked glutamate dehydrogenase in nitrogen metabolism in Saccharomyces cerevisiae. $\mathbf{J}$ Bacteriol 172(9): 4927-4935.

6. Romano $\mathrm{AH}$ and Nickerson WJ (1958). Utilization of amino acids as carbon sources by Streptomyces fradiae. J Bacteriol 75(2): 161-166.

7. Ekena K and Maloy S (1990). Regulation of proline utilization in Salmonella typhimurium: how do cells avoid a futile cycle? Mol Gen Genet 220(3): 492-494.

8. Freese S, Vogts T, Speer F, Schäfer B, Passoth V, and Klinner U (2011). C- and N-catabolic utilization of tricarboxylic acid cycle-related amino acids by Scheffersomyces stipitis and other yeasts. Yeast 28(5): 375-390.

9. Moses S, Sinner T, Zaprasis A, Stöveken N, Hoffmann T, Belitsky BR, Sonenshein AL, and Bremer E (2012). Proline utilization by Bacillus subtilis: uptake and catabolism. J Bacteriol 194(4): 745-758.

10. Nishida I, Watanabe D, Tsolmonbaatar A, Kaino T, Ohtsu I, and Takagi H (2016). Vacuolar amino acid transporters upregulated by exogenous proline and involved in cellular localization of proline in Saccharomyces cerevisiae. J Gen Appl Microbiol 62(3): 132-139.

11. Sickmann A, Reinders J, Wagner Y, Joppich C, Zahedi R, Meyer HE, Schönfisch B, Perschil I, Chacinska A, Guiard B, Rehling P, Pfanner N, and Meisinger $C$ (2003). The proteome of Saccharomyces cerevisiae mitochondria. Proc Natl Acad Sci USA 100(23): 13207-13212.

12. Reinders J, Zahedi RP, Pfanner N, Meisinger C, and Sickmann A (2006). Toward the complete yeast mitochondrial proteome: multidimensional separation techniques for mitochondrial proteomics. J Proteome Res 5(7): 1543-1554.

13. Claros MG and Vincens $P$ (1996). Computational method to predict mitochondrially imported proteins and their targeting sequences. Eur J Biochem 241(3): 779-786.

\section{CONFLICT OF INTEREST}

The authors declare no conflict of interest.

\section{COPYRIGHT}

(C) 2016 Nishida et al. This is an open-access article released under the terms of the Creative Commons Attribution (CC BY) license, which allows the unrestricted use, distribution, and reproduction in any medium, provided the original author and source are acknowledged.

Please cite this article as: Ikuhisa Nishida, Daisuke Watanabe, and Hiroshi Takagi (2016). Putative mitochondrial $\alpha$-ketoglutaratedependent dioxygenase Fmp12 controls utilization of proline as an energy source in Saccharomyces cerevisiae. Microbial Cell 3(10): 522-528. doi: 10.15698/mic2016.10.535

14. García-Campusano F, Anaya VH, Robledo-Arratia L, Quezada $\mathrm{H}$ Hernández H, Riego L, González A (2009). ALT1-encoded alanine aminotransferase plays a central role in the metabolism of alanine in Saccharomyces cerevisiae. Can J Microbiol 55(4): 368-374.

15. Repetto B and Tzagoloff A (1989). Structure and regulation of $K G D 1$, the structural gene for yeast $\alpha$-ketoglutarate dehydrogenase. Mol Cell Biol 9(6): 2695-2705.

16. Moye WS, Amuro N, Rao JK, and Zalkin H (1985). Nucleotide sequence of yeast $G D H 1$ encoding nicotinamide adenine dinucleotide phosphate-dependent glutamate dehydrogenase. J Biol Chem 260(14): 8502-8508.

17. Avendaño A, Deluna A, Olivera $H$, Valenzuela L, and Gonzalez A (1997). GDH3 encodes a glutamate dehydrogenase isozyme, a previously unrecognized route for glutamate biosynthesis in Saccharomyces cerevisiae. J Bacteriol 179(17): 5594-5597.

18. Coleman ST, Fang TK, Rovinsky SA, Turano FJ, and Moye-Rowley WS (2001). Expression of a glutamate decarboxylase homologue is required for normal oxidative stress tolerance in Saccharomyces cerevisiae. J Biol Chem 276(1): 244-250.

19. Avalos JL, Fink GR, and Stephanopoulos G (2013). Compartmentalization of metabolic pathways in yeast mitochondria improves the production of branched-chain alcohols. Nat Biotechnol 31(4): 335341.

20.Spitznagel D, Ebikeme C, Biran M, Nic a' Bháird N, Bringaud F, Henehan GT, and Nolan DP (2009). Alanine aminotransferase of Trypanosoma brucei - a key role in proline metabolism in procyclic life forms. FEBS J 276(23): 7187-7199.

21. Bursell E (1963). Aspects of the metabolism of amino acids in the tsetse fly, Glossina (Diptera). J Insect Physiol 9(4): 439-452.

22. Hausinger RP (2004). Fe(II)/ $\alpha$-ketoglutarate-dependent hydroxylases and related enzymes. Crit Rev Biochem Mol Biol 39(1): 21-68.

23. Strijbis K, van Roermund CW, Hardy GP, van den Burg J, Bloem K, de Haan J, van Vlies N, Wanders RJ, Vaz FM, and Distel B (2009). Identification and characterization of a complete carnitine biosynthesis pathway in Candida albicans. FASEB J 23(8): 2349-2359.

24. Ljungdahl PO and Daignan-Fornier B (2012). Regulation of amino acid, nucleotide, and phosphate metabolism in Saccharomyces cerevisiae. Genetics 190(3): 885-929.

25. Kayikci Ö and Nielsen J (2015). Glucose repression in Saccharomyces cerevisiae. FEMS Yeast Res 15(6): fov068. 
26. Van Mullem V, Wery M, De Bolle X, and Vandenhaute J (2003). Construction of a set of Saccharomyces cerevisiae vectors designed for recombinational cloning. Yeast 20(8): 739-746.
27. Daum G, Böhni PC, and Schatz G (1982). Import of proteins into mitochondria. Cytochrome $b_{2}$ and cytochrome $c$ peroxidase are located in the intermembrane space of yeast mitochondria. J Biol Chem 257(21): 13028-13033. 